\title{
Portrait des Musées d’entreprise en FranCe
}

\author{
Roma LAMBERT ${ }^{1}$
}

L'enjeu médiatique et promotionnel que représente l'exposition du patrimoine a été perçu par un certain nombre d'entreprises depuis le $\mathrm{XIX}^{\mathrm{e}}$ siècle, et certaines d'entre elles se sont lancées dans l'aventure muséale. Ces tentatives restent multiformes et la meilleure façon de rassembler ces « musées d'entreprise » reste une étude expographique de leurs espaces. Sur une étude de 104 musées français, des invariants propres à ce genre muséal ont pu être dégagés sur la base de quatre critères muséographiques et communicationnels, permettant ainsi de dresser le portrait des musées d'entreprise tels qu'ils se présentent actuellement en France.

Depuis le siècle dernier, les entreprises ont pu mesurer la valeur communicationnelle des expositions d'art et d'industrie qui jalonnèrent le $\mathrm{XIX}^{\mathrm{e}}$ siècle. En effet, l'exposition, telle qu'elle est théorisée par André Gob et Noémie Drouguet (Gob \& Drouguet, 2014), possède son propre langage qui s'appuie sur ce qu'elle présente et sur ce qui la constitue, c'est-à-dire les objets donnés à voir aux visiteurs, quels qu'ils soient (œuvre d'art, objet technique, reproduction, maquette), mais aussi les supports de médiation, le mobilier d'exposition (vitrines, meubles, sièges, signalétiques, etc.) et l'architecture du lieu d'accueil si elle est apparente

1 Roma Lambert est diplômée de l'École du Louvre, Paris.

Recherches en communication, ${ }^{\circ} 45$ - Article publié le 23/10/2018 
dans les espaces de l'exposition. Tous ces éléments, ces unités de base du langage, sont désignés par André Desvallées sous le terme d' « expôts » (Desvallées \& Mairesse, 2011).

Le rapport direct aux choses (Davallon, 1999) dans un lieu dédié à leur présentation permet de mettre en place un système de communication tout à fait original. Leur point commun était la présentation d'éléments issus du patrimoine des entreprises qui devenait à cette occasion le support d'un discours spécifique et l'objet d'un regard particulier porté sur l'entreprise.

Néanmoins, un certain nombre de sociétés semble avoir créé un musée de manière quasi accidentelle, ou sans prendre parfaitement conscience de la portée non seulement communicationnelle, mais également scientifique de ce type d'espace. Il en résulte que le paysage des musées d'entreprise tel qu'il se présente actuellement en France est tout à fait hétéroclite, coexiste avec d'autres types d'institutions culturelles et d'offres adressées au public comme la visite d'entreprise. Isabelle Cousserand-Blin fait état des spécificités que ces musées présentent, tout en appuyant sur la complexité du repérage de ces institutions liée à la difficulté de définir ce genre muséal, et à la disparité géographique des lieux identifiés (Cousserand-Blin, 2014). À titre d'exemple, Victor Danilov dénombre seulement 4 musées d'entreprises français en 1991 tandis que Pascale Meyssonat-Courtois en compte 75 en 1994 et qu'Ariane Warlin mentionne 1.500 expériences muséales en 2010. Plus qu'un accroissement exponentiel du nombre de musées, il faut attribuer à la diversité de ces chiffres l'absence de recensement exhaustif des musées d'entreprise et la difficulté à en saisir les spécificités. Isabelle Cousserand-Blin (Cousserand, 2009) propose d'employer le terme « musée d'entreprise » :

« de manière générale lorsqu'une organisation crée et/ou aménage sur son lieu d'activité ou sur un site de production ou à l'extérieur de ceux-ci, un espace muséal, qui présente et met en valeur son passé, ses techniques, son savoir-faire, sa production, ses marques, son secteur professionnel, le territoire sur lequel elle est implantée ; cet endroit consacré lui permet d'accueillir un public spécifique ou hétérogène, restreint ou élargi, en fonction d'objectifs et d'enjeux diversifiés. » 
Et le Dictionnaire encyclopédique de muséologie d'André Desvallées et François Mairesse (2011) propose quant à lui la définition suivante :

« Musée d'entreprise (angl. Company museum, esp. Museo de la empresa). n.m. - Catégorie de musée de nature événementielle et liée à une entreprise encore en activité, destinée à la préservation et la mise en valeur de l'histoire de cette entreprise depuis sa fondation, notamment à partir des produits qu'elle a réalisés. La plupart des grandes entreprises ont développé de tels musées, parfois au bénéfice exclusif de leur personnel - tant pour partager la culture de l'entreprise que servir de réservoir formel, comme ce fut le cas pour les musées de manufactures - parfois également pour le grand public. Le musée, dans ce dernier cas, s'intègre à la stratégie de marketing et de communication de l'entreprise - c'est le cas en France du musée Perrier à Vérèze, du musée du Bonbon Haribo à Uzès, ou en Allemagne du Mercedes-Benz de Stuttgart, etc. »

De ces deux propositions de définitions découlent les mêmes idées de présentation de l'histoire et du savoir-faire d'une société dans un espace en dur permettant d'accueillir physiquement des publics.

Ces deux caractérisations du musée d'entreprise fixent le noyau dur du genre mais sa délimitation - pour quels publics ? avec quels moyens ? selon quels critères de création ? - et les frontières qui le séparent des autres catégories d'espaces muséaux - et surtout des musées publics - restent floues et mouvantes comme en témoigne le statut de certaines entreprises dont le développement est étroitement lié à l'histoire nationale et étatique comme les manufactures réunies au sein du Mobilier national ou encore les sociétés anonymes à capitaux publics comme La Poste ou ENGIE (ex-EDF GDF).

Aussi ai-je souhaité conduire une recherche répondant à la problématique suivante : Existe-t-il ou non une muséographie propre aux musées d'entreprise qui permettrait de les distinguer d'un point de vue visuel, symbolique et communicationnel des musées publics - nationaux ou territoriaux - et même des autres 
types de musées privés, comme les fondations d'entreprise ou les musées de collectionneurs ? Il fallait pour cela se pencher sur les éléments qui fondent la communication verbale et non verbale d'une entreprise via son musée, sur ses buts avoués ou non ainsi que sur les systèmes de langage qu'elle adopte. Dans ce but, une étude comparative a été menée en 2015 sur un corpus de musées se désignant comme appartenant au genre du musée d'entreprise.

Afin de constituer ce corpus, une première liste de musées susceptibles d'entrer dans le champ de la définition de ce qu'est un musée d'entreprise ou bien d'entreprises susceptibles d'avoir créé un musée a été établie grâce à des guides et des répertoires touristiques, des bases de données et des sites Internet dédiés. Après prise de contact avec ces musées et ces entreprises et après affinage du corpus, un questionnaire aménagé a été envoyé à ces structures ainsi ciblées et des entretiens ont été conduits avec certains directeurs ou conservateurs. Ainsi, les musées d'entreprise retenus sont conformes aux définitions mentionnées supra dans la mesure où leur discours porte sur l'histoire, le secteur d'activité ou les savoir-faire et le patrimoine de l'entreprise qui les a créés et qui est toujours en activité. Entrent dans cette catégorie les musées et collections créés par les employés d'une entreprise à titre personnel qui ont par la suite été intégrés à l'organisation de la société (le musée SAFRAN-SNECMA est un très bon exemple de ce cas de figure). À l'inverse, ont été exclus les musées et collections constitués au sein des grandes entreprises nationales ou nationalisées - exception faite des musées d'entreprise publiques aujourd'hui privatisées -, les fondations d'entreprise présentant des collections de nature artistique ou d'une nature qui n'a pas de lien avec l'histoire et la production de l'entreprise, les galeries d'exposition permettant d'accueillir des artistes contemporains.

Les informations ainsi rassemblées m'ont permis de mettre en place un corpus thématisé de 104 musées d'entreprise français, sélectionnés après l'affinage des premières listes et grâce aux réponses des personnes interrogées (tous les responsables des musées d'entreprise entrant dans le corpus n'ont pas répondu à mon appel, loin s'en faut). Notons d'une part que les entreprises à l'origine de ces créations muséales se distinguent par une très 
grande variété. Variété d'échelle tout d'abord, puisque des musées de multinationales cotées au CAC 40 - Hermès International, Michelin, PSA Groupe, Orange - coexistent avec des musées de PME familiales, comptant seulement quelques employés (Chocolaterie Dousset, Faïencerie de Ponchon) ; variété également dans les secteurs d'activités des entreprises - luxe, agroalimentaire, automobile, télécommunication [j'en citerai plein] - bien qu'une prépondérance de certains soit nettement visible. Ce constat peut être imputé à différents facteurs comme l'attractivité présumée du secteur et son potentiel évocateur (ce cas de figure concerne par exemple l'agroalimentaire - la gastronomie française étant perçue comme un patrimoine fortement identitaire - ou encore les secteurs liés à la mode et au luxe); la muséalité des productions du secteur concerné et de son patrimoine en général (il est plus facile d'exposer des produits manufacturés et des packaging comme des bijoux, des moteurs ou des flacons de parfum, que des denrées périssables ou d'évoquer le principe des télécommunication) ; la place accordée aux produits de certains secteurs au sein des musées publics ; les normes sanitaires ou les secrets industriels plus pesants au sein de certains secteurs que d'autres; ou enfin la perception du secteur dans son ensemble par le grand public et les risques de dérives communicationnelles autour du musée (certains projets de musées, comme l'« atelier du médicament » Sanofi à Lyon, n'ont pas vu le jour à cause des inquiétudes des dirigeants des sociétés à cause de ces risques de scandales [Kindermans, 2015]). Ainsi le secteur agroalimentaire est le plus fortement représenté au sein de notre corpus (63 musées sur 104) tandis que les secteurs des télécommunications (1 musée), du bois et du papier (3 musées) et de l'aéronautique (1 musée) sont les moins représentés.

C'est d'après ce corpus qu'il a été possible de faire émerger des invariants constituant les traits les plus caractéristiques du genre muséal qui nous intéressent ici et qui permettent de brosser le portrait des musées d'entreprise tels qu'ils se présentent en France à l'heure actuelle. 


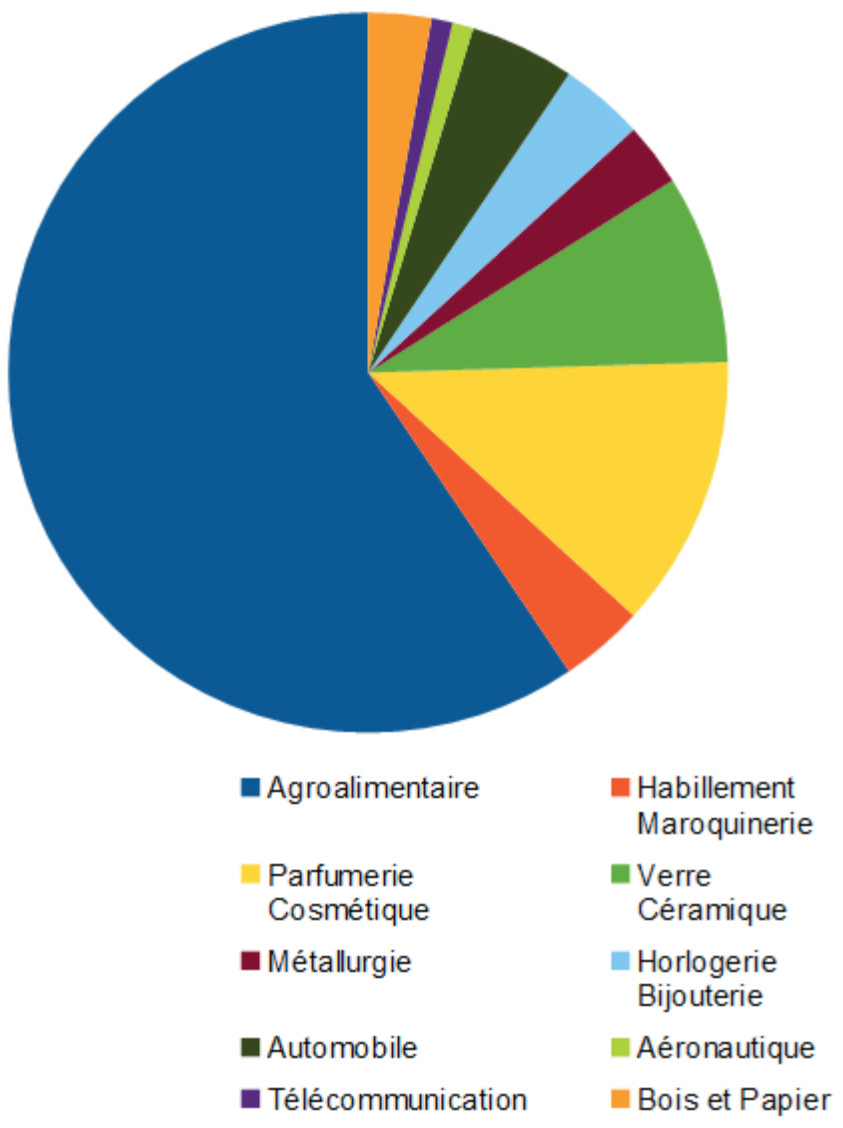

Table 1. Répartition des musées d'entreprise français selon le secteur d'activité de l'entreprise

\section{Quelles spécificités pour les musées d'entreprise?}

Malgré des similitudes formelles dans la nature des objets exposés, Isabelle Cousserand-Blin rappelle qu'il ne faut pas confondre les musées d'entreprise avec des musées de société ou des écomusées. Un musée d'entreprise n'entre pas dans la définition du musée soumise par le Conseil international des Musées (ICOM) dans ses statuts adoptés lors de la $21^{\mathrm{e}}$ Conférence en 
$2007^{2}$. La démarche muséale des entreprises participe directement de leur communication et pour cela leurs musées entrent dans un processus de légitimation et de valorisation au moyen de la présentation du patrimoine et de l'histoire de l'entreprise. Ce but pas toujours avoué du musée créé par une entreprise détermine des choix muséographiques propres au genre et suppose :

- une implantation géographique particulière, en lien direct avec le site de production;

- l'exposition du patrimoine de l'entreprise permettant de développer un certain nombre de thématiques ciblées ;

- une modalité de diffusion du discours proche des habitudes entrepreneuriales.

Selon les propos de Patrice de la Broise (Broise (de la), 2002), " l'entreprise expose (sous la forme de collection propres ou acquises) et s'expose en se rendant visible (par l'architecture) et accessible (par la visite de ses installations) ».

\section{L’implantation géographique}

Sauf exceptions, les musées sont implantés sur le site de production des sociétés ou dans sa proximité immédiate. La répartition des musées d'entreprise sur le territoire français se trouve donc directement soumise à l'histoire des industries en France, elle-même liée aux ressources naturelles qu'offre chaque région.

Le musée en tant qu'architecture est le lieu de sacralisation et de diffusion du discours de l'institution qu'il représente (l'État ou l'entreprise). Son aspect est hautement symbolique et l'implantation choisie par une entreprise pour son musée renseigne immédiatement sur les enjeux communicationnels dont elle pare son espace d'exposition.

2 «Un musée est une institution permanente sans but lucratif au service de la société et de son développement ouverte au public, qui acquiert, conserve, étudie, expose et transmet le patrimoine matériel et immatériel de l'humanité et de son environnement à des fins d'études, d'éducation et de délectation. »

Cette définition fait référence dans la communauté internationale. 


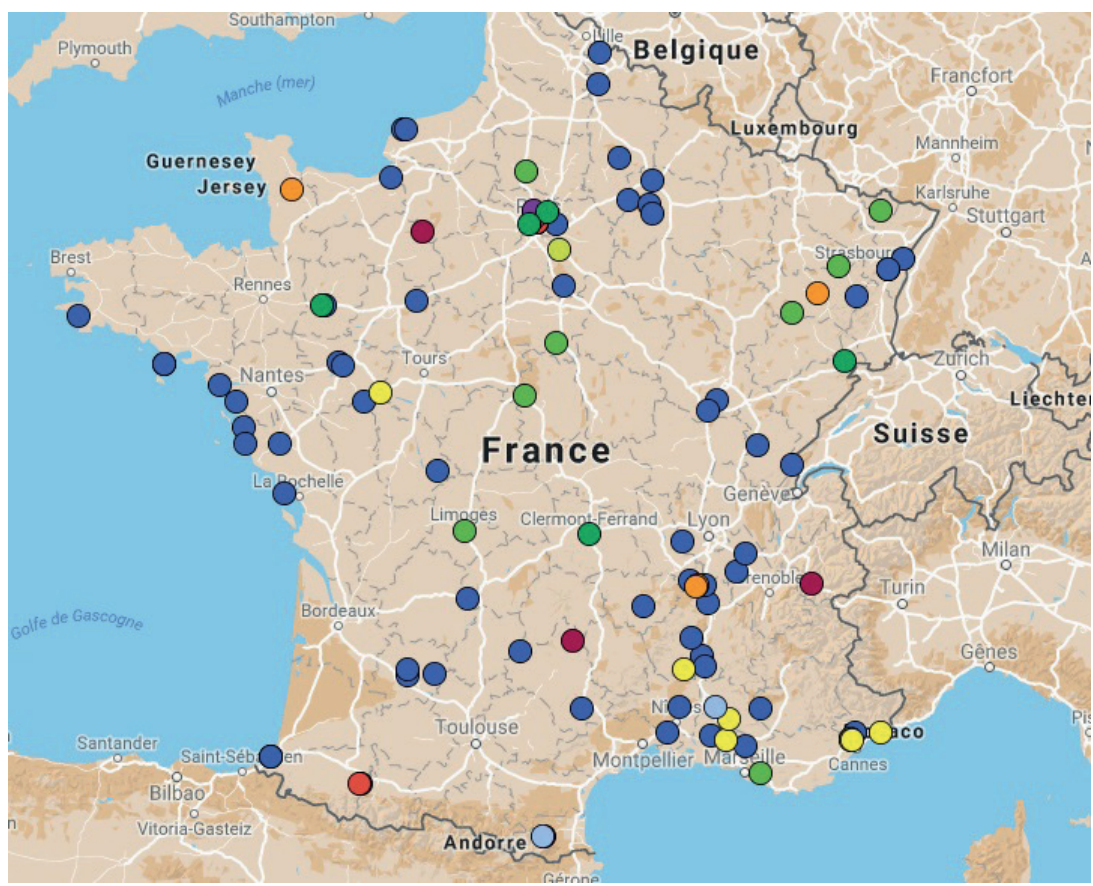

Table 2. Répartition des musées d'entreprise sur le territoire français métropolitaine

Cinq types d'aménagements sont principalement retenus pour les musées d'entreprise :

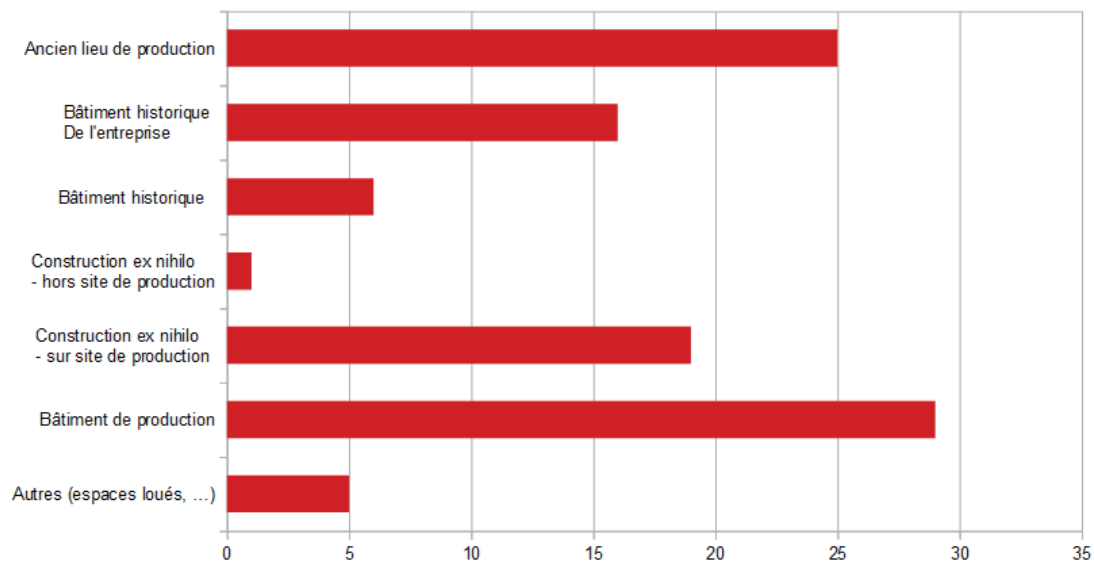

Table 3. Les différents types de bâtiments retenus pour les musées d'entreprise en France 


\section{L'implantation du musée dans l'ancien lieu de production réhabilité pour l'occasion}

L'implantation du musée au sein même d'un édifice marquant soit par son architecture, soit par son ancienneté, est une solution intéressante car elle engage un cercle vertueux : le musée permet de valoriser le patrimoine bâti en lui conférant un statut symbolique et culturel fort, et l'architecture prestigieuse ou frappante de l'édifice sert le discours du musée qui s'en trouve grandi. La pierre, la brique, le fer, le verre qui constituent l'essentiel de ces architectures renvoient immédiatement au travail industriel et sont très évocateurs.

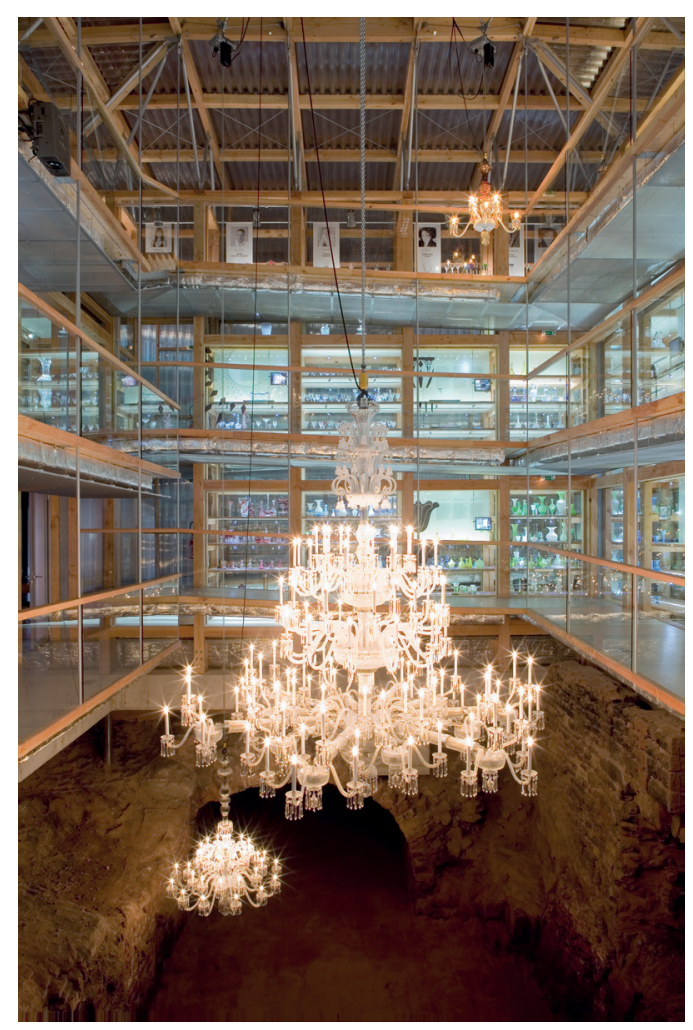

Figure 1. Vue du parcours d'exposition de La Grande Place Musée du Cristal Saint Louis pensé par l'agence architectes Lipsky Rollet (C) Paul Raftery 
Les entreprises choisissent souvent d'anciens bâtiments de production (entrepôts, ateliers, etc.) que les avancées technologiques ont rendu obsolètes. La Grande Place - Musée du Cristal Saint Louis (Saint-Louis-lès-Bitche) illustre un investissement du bâti historique - un ancien four à pots du $\mathrm{XIX}^{\mathrm{e}}$ siècle, situé au centre de la manufacture - qui tire le meilleur parti de l'architecture patrimoniale tout en l'amplifiant au moyen d'une structure moderne.

Si l'entreprise a la chance de conserver des machines ou du mobilier qui deviennent les principaux expôts in situ du nouveau musée, le discours muséal se trouve renforcé par un rapport direct entre le musée comme lieu de médiation et de connaissance, et le site comme lieu d'histoire. Les visiteurs ont le sentiment d'accéder à ce qui a été la « vérité » du travail au sein de l'entreprise.

\section{L'implantation du musée dans un bâtiment historique appartenant à l'entreprise et directement lié à son histoire}

Le musée peut être implanté dans d'anciens lieux de vie : les habitations des ouvriers, ou plus souvent la maison du directeur (16 musées sur 104 retiennent cette solution). Dans ce cadre, le discours porte plus naturellement sur les habitants de ces lieux ; un glissement de la thèse de l'exposition s'opère de l'entreprise vers des figures humaines qui l'incarnent.

C'est le cas de la maison Louis-Vuitton, construite dans l'enceinte des ateliers d'Asnières-sur-Seine, qui, malgré sa muséalisation, conserve un agencement de demeure privée : le mobilier de la famille a été préservé et ont été ajoutées dans chaque salle des malles historiques qui rappellent le métier de malletier de Louis Vuitton.

\section{L'implantation du musée dans un bâtiment remarquable qui n'est pas lié à l'histoire de l'entreprise}

Cette solution, relativement rare (seulement 6 musées exploitent cette possibilité), permet à l'entreprise de bénéficier de l'aura du bâtiment qu'elle choisit pour implanter son musée. Se mêlent ainsi le discours directement lié à l'entreprise et celui por- 
tant sur le prestige ou la particularité historique de l'édifice. La galerie-musée Baccarat (Paris) est implantée dans l'ancien hôtel particulier de Marie-Laure et Charles de Noailles, célèbre pour sa salle de bal et pour les réceptions fastueuses qui y ont été organisées durant l'entre-deux-guerres; cette atmosphère luxueuse fait écho au discours que la cristallerie développe sur son histoire.

\section{L'implantation du musée dans un bâtiment construit à l'occasion de la création du musée lui-même}

Nombre d'entreprises sont contraintes par la disposition de leurs bâtiments et par les normes d'hygiène et de sécurité auxquelles elles sont soumises à envisager la création d'un bâtiment ex nihilo pour recevoir leur musée. Deux possibilités s'offrent alors à elles : implanter leur musée sur le site de production pour pouvoir mettre directement en relation leur processus de fabrication avec les espaces muséaux, ou au contraire, choisir un autre lieu plus en accord avec l'image qu'elles veulent délivrer ou pour se rapprocher de leur public. Il arrive également que, lors d'un déménagement d'une entreprise dans de nouveaux locaux, un espace dédié au musée soit prévu dans les plans de construction. C'est le cas de l'Espace de Visite de la Belle-Iloise, intégré au programme architectural de la nouvelle usine de la société construite en 1994 dans la zone industrielle de Quiberon.

\section{L'implantation du musée dans un local attenant au site de production encore en activité}

À l'inverse de la tendance précédente, des entreprises choisissent d'agrandir leurs locaux pour qu'ils puissent recevoir un espace muséal, ou bien réorganisent leurs lieux de production afin de dédier une ou deux salles à un espace muséographié.

Ces musées présentent des formes et des options de mises en espace extrêmement diversifiées : parfois les espaces muséographiques sont intégrés à la visite des lieux de production en activité, parfois, au contraire, les visiteurs n'ont pas même conscience de pénétrer dans l'enceinte technique. Malgré les grandes disparités 
dans les choix de mise en espace, cette solution est la plus retenue par les entreprises (29 musées sur 104).

Être immédiatement relié à un site de production industrielle en activité est une spécificité majeure de ce genre muséal et une véritable différenciation pour les musées d'entreprise vis-à-vis des musées publics. Si des musées publics comme la Cité de la Céramique - Sèvres et Limoges présentent également cette particularité, ils restent extrêmement minoritaires au sein du paysage muséal français et ce dispositif est justement dû au caractère commercial de l'institution à laquelle ils sont rattachés. Les écomusées, catégorie de musées qui apparaît en France dans les années 1970 et particulièrement attentifs à l'approche interdisciplinaire, participative et souvent militante (Drouguet, 2015) peuvent également être adossés à un atelier de production souvent artisanal mais leur but est différent de celui du musée d'entreprise dans la mesure où il ne s'agit pas de provoquer des retombées d'image ou financières mais bien de revaloriser la culture locale et le territoire. À l'inverse des musées techniques qui ne conservent, selon Philippe Mairot (Mairot, 1991), que des objets sans pouvoir exposer les savoir-faire et les techniques qui nécessitent leur utilisation, les entreprises sont capables à la fois d'exposer les machines et de donner un aperçu de leur fonctionnement en acte.

\section{Les collections propres aux entreprises}

Le patrimoine qu'une entreprise est susceptible d'accumuler prend des formes qui lui sont spécifiques.

- Les bâtiments historiques entrent pleinement dans le patrimoine d'une entreprise et l'on a vu supra qu'il peut être mis en valeur en accueillant le musée. Pour peu que l'édifice ait été modifié au cours du temps, il devient le marqueur tangible de l'évolution de l'entreprise en plus de présenter différents modèles d'architecture industrielle.

- Le matériel de production (outils, machines, machinesoutils, échantillons) et matériel d'usage (cette catégorie d'objets comprend tout le matériel employé par une entreprise dans son activité et qui, éventuellement, la distingue 
de ses concurrentes : les uniformes, les vêtements de travail, la papeterie à en-tête, par exemple).

Qu'il soit ancien ou moderne, le matériel de production représente souvent une part très importante du patrimoine d'une entreprise qui peut reconstituer toute son histoire au travers de ses outils, plus encore si elle en est elle-même à l'origine.

- Les collections d'auvres d'art et les objets à caractère ethnographique. Une entreprise peut posséder des œuvres d'art pour deux raisons différentes.

- Sa production ou les supports publicitaires développés en son sein sont considérés comme entrant dans le champ de l'histoire de l'art. On attribue ce statut aux productions de certaines cristalleries, maroquineries, orfèvreries, etc., ainsi qu'à des supports publicitaires (des affiches, la plupart du temps) réalisés par des artistes. On retrouve d'ailleurs des exemplaires de ces produits et de ces publicités dans des collections d'art publiques comme des affiches

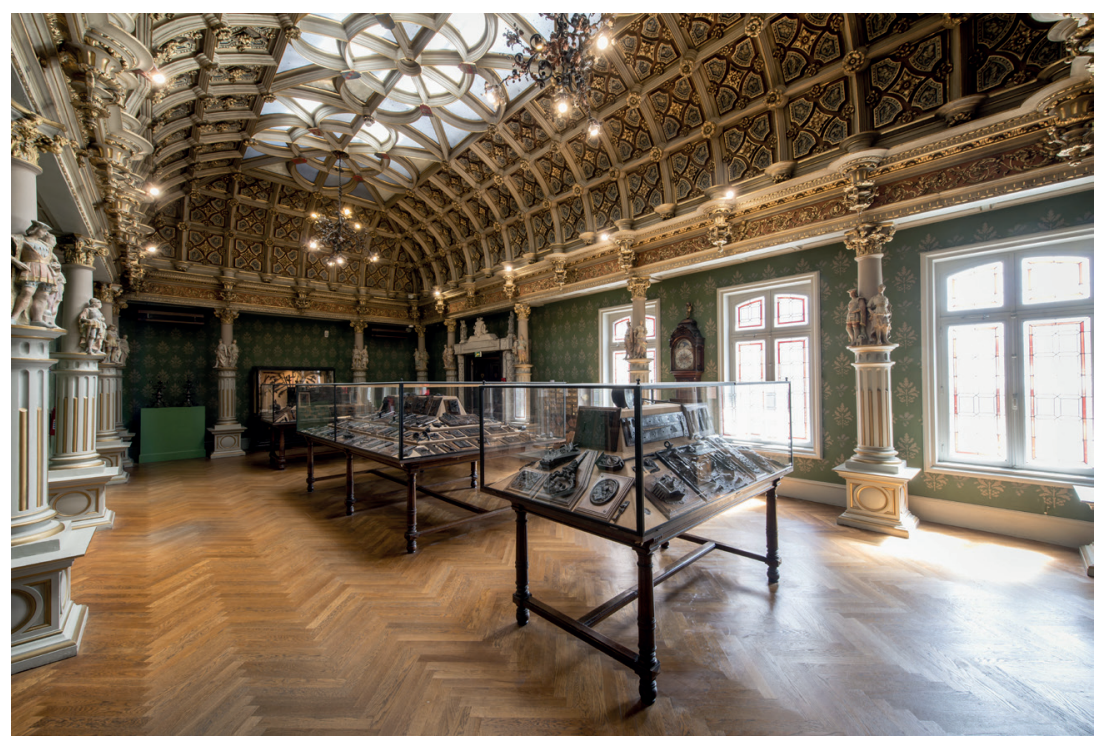

Figure 2. Vue de la salle Renaissance au Palais Bénédictine (C) Palais Bénédictine 
ZAN (ancêtre de Haribo) au musée de Bretagne ou des vases Baccarat au Musée d'Orsay.

- Certaines entreprises ont commandé des œuvres d'art pour embellir leurs locaux ou bien pour renouveler l'image de leur marque. Ces œuvres ont souvent un lien direct avec le secteur d'activité voire les productions de l'entreprise (illustration de l'histoire de l'entreprise, allégorie du produit fabriqué par l'entreprise, etc.). Le Palais Bénédictine (Fécamp) datant des années 1880 abrite aujourd'hui le musée qui bénéficie des acquisitions artistiques réalisées par l'ancien propriétaire : les salles d'exposition mêlent la présentation d'art médiéval, des contenants à liqueurs et des plantes et aromates entrant dans la composition de la Bénédictine.

Il arrive également qu'une entreprise collecte des objets à caractère ethnographique (outils, documents photographiques, costumes, etc.) afin de documenter son secteur d'activité. Elle inscrit ainsi sa pratique dans l'Histoire, ce qui lui confère un prestige ainsi qu'une légitimité dus à l'ancienneté des savoir-faire. Le Lactopôle André Besnier (Laval) a collecté en vue de les exposer

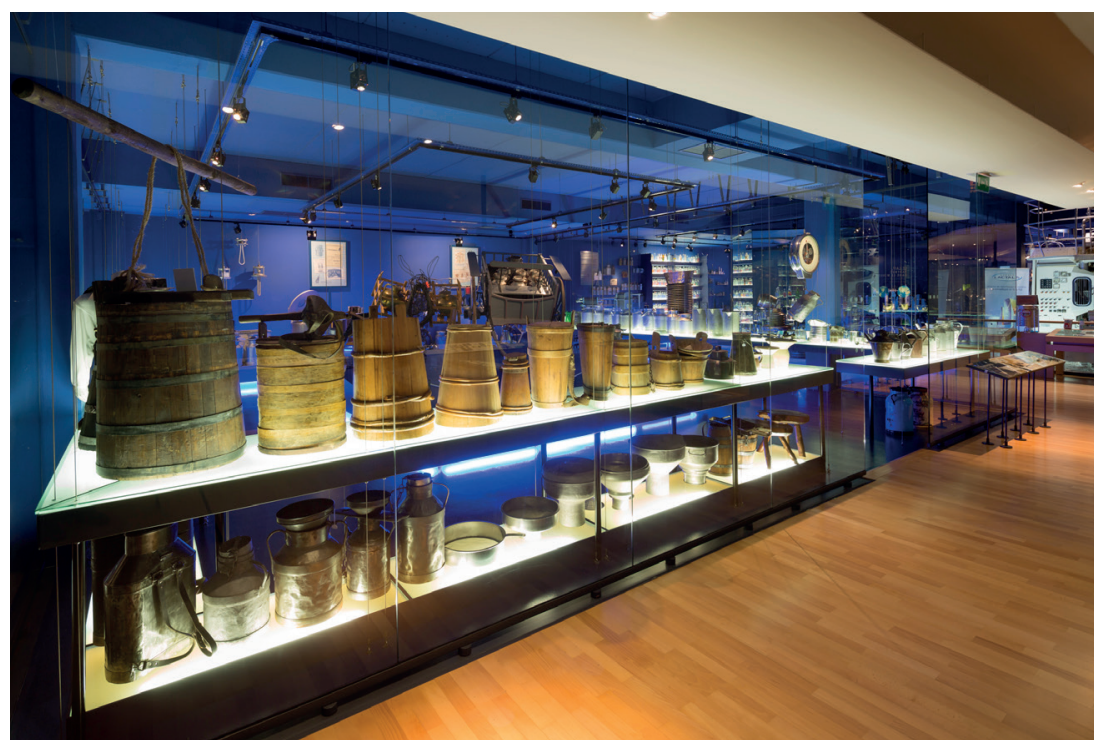

Figure 3. Vue d'une salle muséographique du Lactopôle André Besnier (C) Lactopôle O. DOSSO 
des outils liés à la fabrication de produits laitiers dont l'usage est antérieur à la création de Lactalis.

- Les archives textuelles et photographiques. Certains documents ont une valeur historique et patrimoniale très forte, à l'image des actes de fondations de certaines manufactures. Un fonds iconographique permet également de renseigner l'histoire et d'incarner les personnages fondateurs de l'entreprise.

- Les supports publicitaires et "produits dérivés ». Marqueurs emblématiques, ils sont parfois plus représentatifs de l'activité de l'entreprise que ses productions ellesmêmes. Du fait de la nature périssable de leurs productions, un grand nombre d'entreprises fabriquant des produits alimentaires font ce choix d'expôts.

- Les productions et packagings de l'entreprise restent son premier patrimoine dans la mesure où ils témoignent de son existence et de son activité. Ils ne deviennent exposables qu'à partir du moment où ils perdent leur statut d'objets mis en vente (actifs circulants) pour devenir des témoins de la production de l'entreprise à un moment donné.

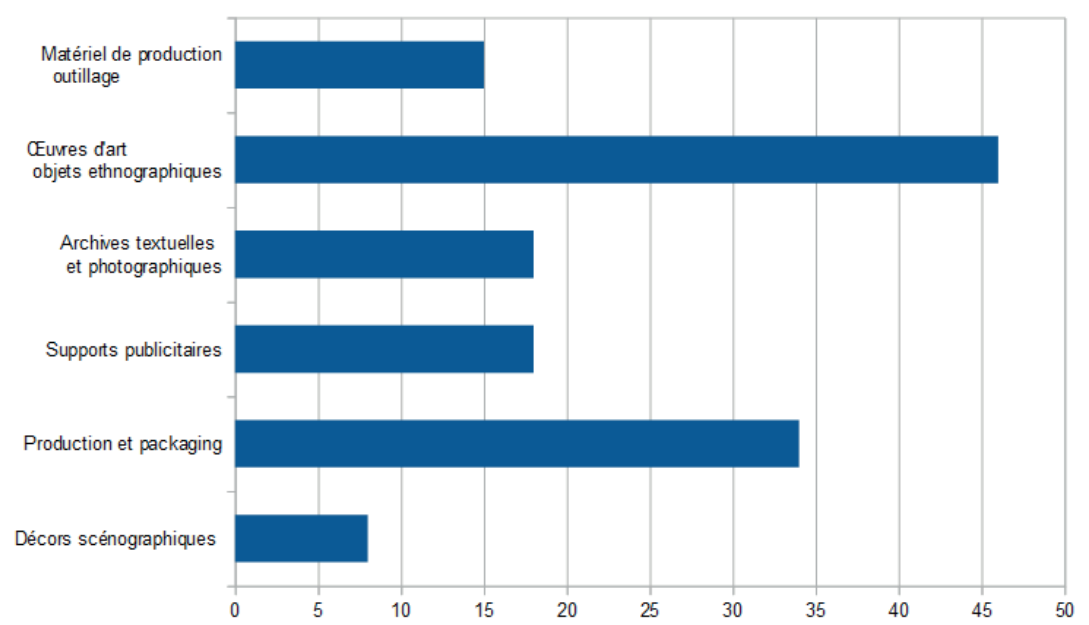

Table 4. Sélection des expôts selon leur nature au sein des musées d'entreprise français 
- Les savoir-faire (know-how). On comprend dans cette catégorie de biens les éléments quantifiables (brevets, marques, etc.) et le patrimoine immatériel de l'entreprise ainsi que la renommée de l'entreprise. Au sein du musée, ce type de patrimoine non exposable en tant que tel se trouve incarné par tous les biens précédemment cités. Ainsi le matériel de production entre dans la catégorie des objets qui sont des truchements analogiques ou métaphoriques, des outils d'exécution qui n'ont de valeur historique, culturelle ou pédagogique que grâce à leur capacité à expliciter un processus, une manipulation, notamment au moyen d'une démonstration (Jadé, 2006, p.1 70).

Les entreprises exposent généralement plusieurs de ces catégories d'objets au sein de leur espace muséal afin de développer un discours riche. Pour que le musée participe de l'image de marque cultivée par l'entreprise, il lui faut parvenir à donner une impression de transparence dans le choix de ses expôts tout en ne montrant que ce qui est valorisant. Ainsi, le choix des entreprises se porte généralement sur 1) des objets-témoins, c'est-àdire les objets qui attestent non seulement des techniques et des savoir-faire à l'œuvre dans l'entreprise, mais également des étapes de son évolution (le matériel de production, certains documents d'archives, le matériel d'usage, certains produits de l'entreprise) ; 2) sur les objets-symboles ou les objets-reliques, objets particulièrement lourds de sens qui peuvent prétendre résumer toute la culture ou toute une époque de l'entreprise. Il s'agit ici d'un certain type de matériel d'usage, de certains supports publicitaires et produits prestigieux, exceptionnels ou au contraire emblématiques de toute la production de l'entreprise, des documents d'archives comme un acte de fondation ou un dépôt de brevet; enfin 3) des objets de mémoire, comme des effets intimes de personnages qui ont marqué l'histoire de l'entreprise, des stylos avec lesquels ont été signés des accords, etc. 


\section{La diffusion de discours de l'entreprise à partir de l'espace muséographique}

\section{A. Les modalités de diffusion de discours}

Au sein de l'exposition, le discours s'appuie essentiellement sur la présentation des objets et de la création de sens qui découle de leur rapprochement, mais il peut également être délivré au moyen de médiation textuelle ou de médiation orale. Au sein d'un musée d'entreprise, les panneaux de textes sont rares, tout autant que les cartels, et, lorsqu'ils sont présents, leur contenu est relativement pauvre de sens. Comme l'analyse Pascale Meyssonat-Courtois (Meysonnat-Courtois, 1994), l'objet n'est très souvent exposé que pour sa valeur esthétique ou sensationnelle, le cartel ne fait que le désigner et ne le renseigne pas. On pourrait penser qu'il s'agit d'un choix délibéré qui découle d'une culture visuelle propre à l'entreprise, et notamment du domaine de la publicité, qui place l'image avant le texte dans l'ordre des signifiants. Les « vraies choses » (productions de l'entreprise, supports publicitaires, machines et outils) restent les supports privilégiés du discours muséographique. Mais cela peut également être dû à la conception de la visite comme un moment de récréation et de ressenti émotionnel qui ne doit pas être rendu rébarbatif par la lecture de panneaux de salle. Cette absence de discours au niveau des objets a toujours deux conséquences selon Pascale Meyssonnat-Courtois : 1) les objets forment un ensemble qui consacre l'espace muséal comme un tout unique dont les expôts sans message particulier ne font que définir le lieu comme musée : le musée est musée quoiqu'il expose, il lui suffit d'exposer pour être, 2) l'objet technique, le produit, la machine se trouvent esthétisés par cette absence de discours qui se trouve ailleurs, dans les commentaires des guides ou des conservateurs qui animent la visite.

En réalité, la visite guidée est bien souvent privilégiée à la médiation écrite et à l'audioguide et ce pour plusieurs raisons. Les personnes en charge de la visite guidée font très souvent partie du personnel de l'entreprise (actif ou retraité) et, en endossant à la fois un rôle d'expert et de témoin, ils maîtrisent symboliquement 
le discours. Mais ces guides sont aussi présents pour encadrer physiquement et temporellement les visiteurs afin de respecter les normes d'hygiène et de sécurité en cas de passage par les ateliers de production. Ils permettent surtout d'assurer des durées de visite à la fois acceptables pour les visiteurs et rentables pour les concepteurs et de s'assurer que le balisage, comprenant la boutique, sera respecté dans sa totalité.

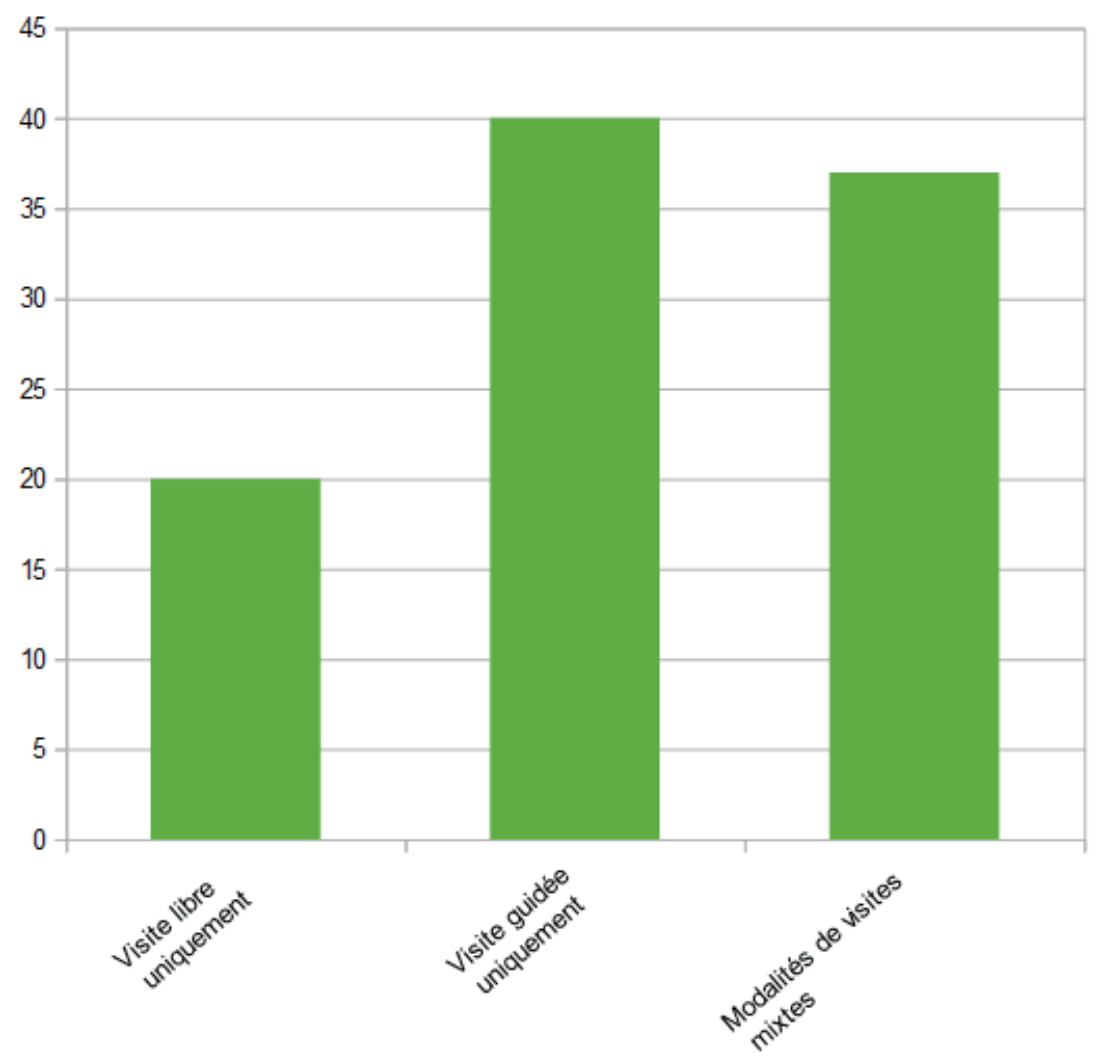

Table 5. Les modalités de visites exploitées dans les musées d'entreprise en France

Néanmoins, les modalités de la visite guidée diffèrent énormément d'un musée à l'autre, aussi bien dans les formes que dans les intentions. Deux exemples diamétralement opposés : 1) $\mathrm{Au}$ musée de la Collection historique Orange, le visiteur prend ren- 
dez-vous et il est reçu en personne par le responsable de la Collection historique, qui, avant d'occuper ce poste, a exercé différents métiers au sein de l'entreprise. 2) Chez Fragonard Parfumeur, les guides ne sont pas choisis pour leurs connaissances historiques de la parfumerie ou de la maison Fragonard mais pour leurs aptitudes linguistiques afin d'accueillir au mieux des groupes de touristes étrangers en visite à Paris. La succession rapide des groupes et la «standardisation » des hôtesses par le port de l'uniforme ne permet pas d'incarner proprement l'esprit de la marque. La visite du musée perd en authenticité ce qu'elle gagne en canalisation du public. Ainsi, la médiation orale, de personne à personne, aide à incarner le discours et à rendre la visite plus vivante et l'expérience imprègne davantage la mémoire du visiteur.

La spécificité du musée d'entreprise par rapport au musée public ne se situe donc pas tant dans le choix des expôts que dans la nature de son discours et dans la manière de le délivrer.

\section{B. Les thématiques propres au musée d'entreprise}

Isabelle Cousserand-Blin (Cousserand-Blin, 2014) rappelle que « le musée d'entreprise offre une occasion unique de rencontre qui permet souvent d'appréhender l'entreprise dans une totalité et dans une continuité ». De fait, les entreprises développent au sein de leurs espaces muséaux un discours propre à présenter ce qu'elles aimeraient que le public découvre et retienne et toutes développent des thématiques très proches liées directement à leur positionnement au sein de son secteur d'activité présent, passé et à venir, et sur leur manière de développer leur production. D'une entreprise à l'autre les musées permettent ainsi de persuader le visiteur de l'excellence des savoir-faire et des produits de l'entreprise, démonstration s'appuyant sur un nombre de thématiques relativement réduites :

- l'histoire du produit : comment a-t-il été inventé ?;

- l'histoire du processus de fabrication : comment a-t-il été mis au point, quelles évolutions successives a-t-il connu pour prendre sa forme actuelle?; 
- l'histoire de l'entreprise : quelles en sont les grandes figures fondatrices, comment l'entreprise a-t-elle surmonté les périodes de crises?;

- l'histoire des fondateurs de l'entreprise : quel est leur parcours personnel, comment ont-ils contribué à leur échelle à la construction de l'histoire nationale ou internationale?;

- l'histoire de la région dans laquelle se trouve implantée l'entreprise ;

Ces thématiques permettent d'une part de développer un discours valorisant tout en délivrant un contenu historique et scientifique pointu, et d'activer d'autre part des outils d'évocation promouvant l'entreprise qui sont :

- Le prestige («nous seuls en sommes capables ») : prestige acquis par l'entreprise, que ce soit grâce à l'ingéniosité et au talent de la figure fondatrice, grâce à la qualité de ses productions et aux figures-phares qui ont traversé l'histoire de l'entreprise en tant que fournisseurs, collaborateurs ou clients.

- La tradition (« nous avons toujours procédé de cette façon ») : liée à cette notion de tradition, l'ancienneté de l'entreprise est présentée, au sein des musées, comme un gage de qualité jamais démenti.

- La nostalgie et la projection régressive (« c'était mieux avant ») : ce procédé entend raviver des sensations et des émotions chez le visiteur, usager de la marque au quotidien.

Au sein de l'Aventure Michelin, le dernier espace du musée est dévolu à la présentation des innovations prestigieuses de l'entreprise Michelin. Sous le titre « Développement et mobilité durables ", cette salle présente au public trois actions récentes de Michelin en faveur de la mobilité durable et notamment la présentation du concept de la roue VISION Michelin ; un moyen d'inscrire l'entreprise dans le présent voire de présenter son futur proche, grâce à un discours mélioratif fondé sur l'innovation technique prestigieuse. 


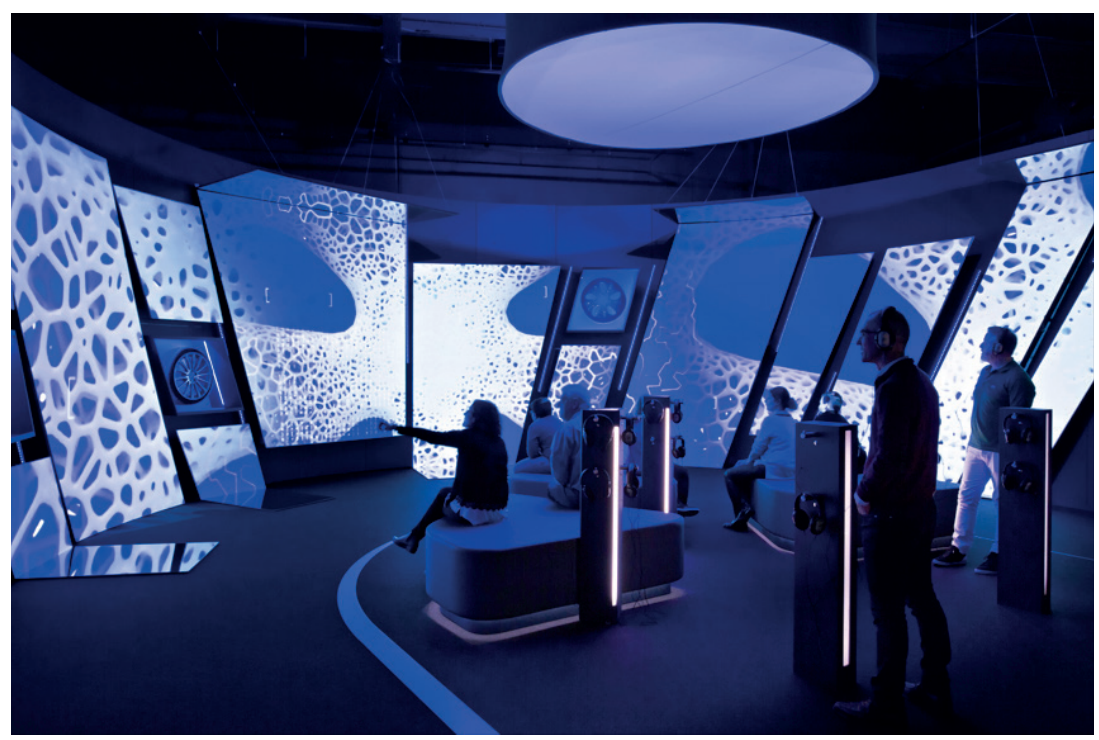

Figure 4. Espace « Développement et mobilité durables 》 de l'Aventure Michelin $@$ Aventure Michelin

La somme de ces angles d'attaque, dispositifs d'évocation et thématiques peuvent être cumulatifs et tous ces outils ont pour but de susciter l'admiration chez le visiteur. Ce dernier, en procédant à l'acte d'achat soit dans la boutique du musée soit plus tard, a le sentiment de bénéficier personnellement du prestige de la marque par la possession d'un produit de l'entreprise. Il s'agit là, somme toute, de l'élément différenciant le plus important du musée d'entreprise vis-à-vis du musée public.

Ces éléments expographiques sont à manipuler avec précaution tant la frontière entre le discours scientifique et objectif et le discours marketing et hagiographique est fine. Certains musées basculent irrémédiablement du côté de la promotion par le recours à un vocabulaire trop proche - voire directement issu - du monde publicitaire. Certains éléments difficiles de l'histoire de l'entreprise - scandales financiers, épisodes de grèves... - peuvent par ailleurs être passés sous silence, traduisant un manque de transparence au profit d'un discours lissé. Le recours à certain type d'expôts comme des films promotionnels utilisés pour délivrer un message scientifique représente la partie la plus visible de ces dérives 
communicationnelles mais il arrive bien souvent que le basculement marketing s'effectue subtilement au détour d'une phrase, par le choix d'un mot, ou par la mise en regard d'un objet marketing avec d'autres expôts : c'est le cas du Musée-Manufacture du Chocolat de la Chocolaterie Sève - musée au discours scientifique par ailleurs très rigoureux sur l'histoire du chocolat - qui présente, au sein d'une vitrine consacrée au cacao comme monnaie d'échange dans le monde rassemblant des objets ethnographiques d'Amérique du Sud, des maquettes de bateaux et des interprétations de gravures du $\mathrm{XVIII}^{\mathrm{e}}$ siècle, des produits de l'entreprise dans leur packaging commercial. Ces dérives, délibérées ou simplement maladroites, peuvent entraîner des réactions parfois virulentes de la part des visiteurs - en témoignent les commentaires postés sur des sites Internet de tourisme comme TripAdvisor - et nuisent à l'image du musée. Un juste équilibre doit donc impérativement être trouvé entre objectivité scientifique et promotion communicationnelle de l'entreprise au sein de l'exposition sous peine de rompre le contrat tacite qui lie les concepteurs du musée et le visiteur en attente d'une approche non communicationnelle de l'entreprise. Certains musées, comme l'Aventure Michelin ou le musée SAFRAN-SNECMA y parviennent avec brio.

\section{Conclusion}

Malgré son ancienneté, le musée d'entreprise semble être encore à un stade d'expérimentation et l'on assiste même à des échecs muséaux : certains musées ont fermé pour de multiples causes, la principale étant qu'ils ne remplissaient pas le rôle que leur avaient assigné leurs concepteurs. Le musée d'entreprise est conçu pour apporter de la richesse à l'entreprise : de la richesse en termes de retombées financières, mais surtout de la richesse en termes d'images, ce qu'aucune campagne publicitaire ne peut faire à l'échelle d'un espace muséal.

La particularité la plus forte du musée d'entreprise d'un point de vue muséographique réside dans l'immédiateté de la production de discours vis-à-vis du sujet présenté dans le musée : une chaîne sémantique ininterrompue se forme entre les ateliers de 
productions et les espaces d'expositions, sans place pour le recul qui existe dans un musée public développant un discours sur un sujet qui est étranger à sa propre histoire et son fonctionnement. De fait, la question de l'actualisation des informations délivrées au sein de l'espace muséal devient donc, de manière corrélative, un enjeu essentiel du fonctionnement à long terme de ce genre muséal qui, très vite, peut présenter un discours obsolète ou une muséographie dépassée par l'histoire récente de la société (fusion, innovations technologiques, nouvelle orientation de la production, etc.).

La constitution d'un portrait véridique du musée d'entreprise en France demeure encore problématique en raison des zones de flou, des expérimentations multiples et du rapport même que les entreprises entretiennent avec la sphère patrimoniale et muséale. Néanmoins, la muséographie d'entreprise existe bel et bien en tant que genre, avec ses codes et ses outils de communication propres, mais elle est d'une plasticité telle que ses limites restent encore mouvantes. Une professionnalisation plus grande de la démarche d'exposition permettrait de tirer des bénéfices nettement supérieurs en termes de communication.

\section{Références}

Broise de la, P. (2002). Esthétique et mises en scène de patrimoine industriel architectural. Recherches en Communication 18, 37-54. Disponible à : http://sites.uclouvain. be/rec/index.php/rec/article/view/3381.

Cousserand, I. (2009). Musées d'entreprise : un genre composite. Communication et organisation 35, 193-213. Disponible à : http://communicationorganisation.revues. org $/ 829$.

Cousserand-Blin, I. (2014). La mosaïque des musées d'entreprise. Quels I pour quelles cultures? Bulletin Amecsti 40, 7-10.

Danilov, V. J. (1991). Corporate Museums, Galleries, and Visitor Centers: A Directory. New York: Greenwood Press.

Davallon, J. (1999). L'exposition à l'œuvre. Paris : L'Harmattan.

Desvallées, A. \& Mairesse, F. (Éd.) (2011). Dictionnaire encyclopédique de muséologie. Paris : Armand Colin.

Drouguet, N. (2015). Le musée de société : De l'exposition de folklore aux enjeux contemporains. Paris : Armand Colin.

Gob, A. \& Drouget, N. (2014). La Muséologie. Histoire, développements, enjeux actuels, 4 édition, coll. Sciences Humaines et Sociales. Paris : Armand Colin. 
Jadé, M. (2006). Patrimoine immatériel. Perspectives d'interprétation du concept de patrimoine. Paris : L'Harmattan.

Kindermans, M. (2015). Les musées d'entreprise ont le vent en poupe. Les Échos. Disponible à : https://business.lesechos.fr/directions-marketing/marketing/ branding/0204083520338-marques-les-musees-d-entreprise-ont-le-vent-enpoupe-107121.php.

Mairot, P. (1991). Musée et technique. Terrain 16, 131-138.

Meyssonat-Courtois, P. (1994). Les musées d'entreprise en France. Une réalité à dépasser, un concept à inventer. La lettre de l'OCIM 34 (3), 13-17.

Statuts de l'ICOM, adoptés lors de la $21^{\text {e }}$ Conférence générale à Vienne en 2007. Disponible à : http://icom.museum/la-vision/definition-du-musee/L/2/).

Warlin, A. (2010). Musée d'entreprise - Un retour sur investissement plus immatériel que matériel. Le nouvel Économiste. Disponible à : http://www.lenouveleconomiste.fr/lesdossiers/musee-dentreprise-un-retour-sur-investissement-plus-immateriel-que-materiel-6386/.

\section{@) $(\Theta \Theta \Theta$}

«Attribution - Pas d'Utilisation Commerciale - Pas de Modification 4.0 International» (CC BY-NC-ND) 